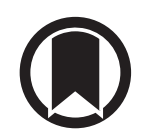

CrossMark

\title{
Guiding antibiotic treatment with inflammatory biomarkers in COPD? Another brick in the wall
}

\author{
Philipp Schuetz ${ }^{1,2}$ and Daiana Stolz ${ }^{3}$ \\ Affiliations: ${ }^{1}$ Dept of Internal Medicine, Kantonsspital Aarau, Aarau, Switzerland. ${ }^{2}$ University Basel, Basel, \\ Switzerland. ${ }^{3}$ Clinic of Respiratory Medicine and Pulmonary Cell Research, University Hospital of Basel,
} Basel, Switzerland.

Correspondence: Philipp Schuetz, Medizinische Universitätsklinik, Kantonsspital Aarau Tellstrasse H7, $\mathrm{CH}-5001$ Aarau, Switzerland. E-mail: schuetzphagmail.com

@ERSpublications

More studies are needed to guide antibiotic treatment with inflammatory biomarkers in COPD http://ow.ly/bJcJ30oklR2

Cite this article as: Schuetz P, Stolz D. Guiding antibiotic treatment with inflammatory biomarkers in COPD? Another brick in the wall. Eur Respir J 2019; 53: 1900562 [https://doi.org/10.1183/13993003.00562-2019].

Acute exacerbations of chronic obstructive pulmonary disease (COPD) often prompt initiation of empirical antibiotic treatment, although in many cases a bacterial pathogen cannot be detected, and viruses may indeed account for a large proportion of episodes. Indeed, data supporting the effectiveness of the broad antibiotic utilisation at exacerbation not requiring intensive care are insufficient. Herein, personalising antibiotic treatment based on a patient's individual risk for bacterial infection has great potential to improve antibiotic stewardship efforts to encourage judicious and correct usage of these agents and mitigate the emergence of multidrug-resistant pathogens, one of the most urgent threats to global health and directly linked to antibiotic overuse. Integration of host response markers, which correlate with bacterial infection, into the overall assessment and clinical care of patients with acute exacerbation of COPD has high potential to improve individual antibiotic decisions. Among such promising host response markers in acute exacerbation of COPD, procalcitonin (PCT), a marker specific to bacterial infections, and C-reactive protein (CRP), a more general inflammatory marker with high sensitivity, have generated most interest. Several randomised trials have found PCT to result in a significant reduction of antibiotic usage with a similar resolution of clinical symptoms in patients with sepsis and respiratory infections, including acute exacerbation of COPD [1, 2]. In fact, a meta-analysis based on individual data of 1252 patients with COPD exacerbation found PCT guidance to result in a significant reduction in antibiotic initiation ( $72 \%$ versus $43 \%)$ and antibiotic exposure (5.3 versus 3.1 days) with no difference in mortality ( $4 \%$ versus $3 \%$ ) or risk of treatment failure (17\% versus 17\%) [3]. Still, a recent trial investigating COPD patients needing intensive care treatment did not report a significant effect of PCT on antibiotic usage, and non-inferiority of PCT in regard to clinical outcomes could not be demonstrated [4]. CRP has been used successfully to direct antibiotic treatment in primary care studies [5, 6], and observational research suggested CRP may be more suitable to direct antibiotic treatment in acute exacerbation of COPD compared to PCT [7]. Still, randomised trials looking at the effect of CRP for guiding antibiotic decisions in patients with acute COPD exacerbation have been largely missing.

In this issue of the European Respiratory Journal, Prins et al. [8] report the result of a trial in 220 patients with acute exacerbation of COPD, comparing antibiotic usage according to a CRP-based strategy with a strategy based on Global Initiative for Chronic Obstructive Lung Disease (GOLD) guidelines. Subjects 
assigned to the CRP group were treated with antibiotic for 7 days if CRP on admission or after $24 \mathrm{~h}$ was $\geqslant 50 \mathrm{mg} \cdot \mathrm{L}^{-1}$. Subjects in the GOLD group were also treated with the same antibiotic regimen if they reported increased sputum purulence in combination with increased dyspnoea and/or increased sputum volume, as propagated by the GOLD guidelines. The results showed a significant reduction in antibiotic initiation of more than $30 \%$ (from $46.2 \%$ to $31.7 \%$ ) associated with the CRP algorithm. There was no difference in clinical outcomes in the short term and after 1-year of follow up, yet the study did not have the statistical power to assess safety of this approach and type II error is possible. Also of note, in 40 patients who were not treated initially, antibiotics were prescribed due to treatment failure. This deviation from the protocol, however, was well-balanced between groups.

Decisions regarding antibiotic use in an individual patient with acute exacerbation of COPD is complex and should be based on several considerations including the pretest probability for bacterial infection, which is based on clinical examination, severity of the disease and results from microbiological tests, the severity of acute episode and results of host response markers [9]. In the context of a low risk situation and a low pretest probability for bacterial infections, a low level of host response markers aids in ruling out bacterial infection and empirical antibiotic therapy can be avoided. In some patients, this strategy may not play out and clinical assessment and retesting is mandatory. While there was data on the use of PCT to direct antibiotic decisions from several trials, PRINs et al. [8] have now provided important study results implying that CRP may also be helpful to direct treatment. While results are most convincing regarding the lowering of antibiotic exposure, proof of safety using CRP is still pending. Ultimately, a head to head trial will be needed to compare a CRP versus a PCT strategy taking into account effects on antibiotic treatment, patient outcomes and costs [6].

There is a strong interest in the medical community in reducing unnecessary antibiotic exposure. The urgency in rationalising and individualising antibiotic decisions is particularly meaningful in a situation in which antibiotics can be withheld in the vast majority of the cases, such as for exacerbations of COPD [10]. Integration of host response markers into treatment decision, now also including CRP, has shown promising results. These markers, however, should not be used as a substitute for good clinical practice, but rather be part of the overall assessment of a patient. Decisions pertaining to the initiation and cessation of antibiotic treatment remains strongly dependent on an assessment of all available clinical and diagnostic parameters, including a thorough patient assessment and the severity of the illness. Still, host response markers remain the best line of defence against diagnostic uncertainty and antibiotic overuse. Further research is needed to compare markers head to head, and to explore their optimal combination with pathogen-directed tests.

Conflict of interest: P. Schuetz reports grants (paid to institution) from bioMerieux, Thermofisher and Roche Diagnostics, outside the submitted work. D. Stolz has received research grants from the Swiss National Foundation, AstraZeneca AG, Pan Gas AG, Weinmann AG, Curetis AG, Boston Scientific AG, Circassia Pharmaceuticals and Lungenliga Switzerland; received payment for lectures, advisory panels or sponsorship to attend scientific meetings from Novartis AG, AstraZeneca AG, GSK AG, Roche AG, Zambon, Pfizer and Schwabe Pharma AG; is the current education council chair of the European Respiratory Society and immediate past president of the European Board of Accreditation in Pneumology, and is the current GOLD representative for Switzerland.

\section{References}

1 Stolz D, Christ-Crain M, Bingisser R, et al. Antibiotic treatment of exacerbations of COPD: a randomized, controlled trial comparing procalcitonin-guidance with standard therapy. Chest 2007; 131: 9-19.

2 Wirz Y, Meier MA, Bouadma L, et al. Effect of procalcitonin-guided antibiotic treatment on clinical outcomes in intensive care unit patients with infection and sepsis patients: a patient-level meta-analysis of randomized trials. Crit Care 2018; 22: 191.

3 Schuetz P, Wirz Y, Sager R, et al. Effect of procalcitonin-guided antibiotic treatment on mortality in acute respiratory infections: a patient level meta-analysis. Lancet Infect Dis 2018; 18: 95-107.

4 Daubin $\mathrm{C}$, Valette $\mathrm{X}$, Thiolliere $\mathrm{F}$, et al. Procalcitonin algorithm to guide initial antibiotic therapy in acute exacerbations of COPD admitted to the ICU: a randomized multicenter study. Intensive Care Med 2018; 44: 428-437.

5 Cals JW, Butler CC, Hopstaken RM, et al. Effect of point of care testing for C reactive protein and training in communication skills on antibiotic use in lower respiratory tract infections: cluster randomised trial. BMJ 2009; 338: b1374.

6 Meili M, Muller B, Kulkarni P, et al. Management of patients with respiratory infections in primary care procalcitonin, C-reactive protein or both? Expert Rev Respir Med 2015; 9: 587-601.

7 Daniels JM, Schoorl M, Snijders D, et al. Procalcitonin vs C-reactive protein as predictive markers of response to antibiotic therapy in acute exacerbations of COPD. Chest 2010; 138: 1108-1115.

8 Prins HJ, Duijkers R, van der Valk P, et al. CRP-guided antibiotic treatment in acute exacerbations of COPD in hospital admissions. Eur Respir J 2019; 53: 1802014.

9 Neeser O, Branche A, Mueller B, et al. How to: implement procalcitonin testing in my practice. Clin Microbio Infect 2019; in press [https://doi.org/10.1016/j.cmi.2018.12.028].

10 Van Velzen P, Ter Riet G, Bresser P, et al. Doxycycline for outpatient-treated acute exacerbations of COPD: a randomised double-blind placebo-controlled trial. Lancet Respir Med 2017; 5: 492-499. 